\title{
From Centralized to Distributed Sensor Localization
}

\author{
Amin Karbasi \\ LTHC \& LCAV \\ School of Compter and Communication Sciences \\ EPFL, Switzereland \\ amin.karbasi@epfl.ch
}

\begin{abstract}
In this work we consider the problem of sensor network localization when only the connectivity information is available. More specifically, we compare the performance of the centralized algorithm MDS-MAP with its distributed version HOP-TERRAIN. We show that both algorithms are able to localize sensors up to a bounded error decreasing at a rate inversely proportional to the radio range $r$.
\end{abstract}

\section{Categories and Subject Descriptors}

C.2.1 [Computer Systems Organization,]: ComputerCommunication Networks, Network Architecture and Design [Wireless Communication]

\section{General Terms}

Theory, Performance, Algorithms

\section{Keywords}

localization, sensor network

\section{INTRODUCTION}

This problem of localization plays an important role in wireless sensor networks when the positions of the nodes are not provided in advance. One costly way to find the positions is by equipping all the sensors with a global positioning system (GPS). Apart from its considerable cost to the system, the major drawback of such a systems is that it does not work in indoor environments. An alternative way is to acquire the positions of sensors based on basic local information such as proximity or local distances.

Recently, a great deal of work focused on localization algorithms for sensor networks [9, 6, 5, 8, 11, 1, 12, 13]. Based on the approach of processing the distance measurements, these algorithms can be classified into two categories: centralized algorithms and distributed algorithms. In centralized algorithms $[12,1]$, all the distance measurements are sent to a single processor where the estimated positions are computed.

Permission to make digital or hard copies of all or part of this work for personal or classroom use is granted without fee provided that copies are not made or distributed for profit or commercial advantage and that copies bear this notice and the full citation on the first page. To copy otherwise, to republish, to post on servers or to redistribute to lists, requires prior specific permission and/or a fee.

S3'10, September 20, 2010, Chicago, Illinois, USA.

Copyright 2010 ACM 978-1-4503-0144-2/10/09 ...\$10.00.
On the other hand, in distributed algorithms $[9,6,5,8,11$, $13]$ there is no central infrastructure and nodes through message passing with their local neighbours share information. This way they collectively estimate their pairwise distances and compute their own positions.

The performance of these practical algorithms are mainly measured through simulations. Of the same interest and complementary in nature, however, are the theoretical guarantees associated with the performance of the existing methods. Such analytical bounds on the performance of localization algorithms can provide answers to practical questions: for example, how large should the radio range be in order to get the error within a threshold? With this motivation in mind, our work takes a forward step in this direction. In particular, we provide and compare the performance bounds of the centralized algorithm MDS-MAP [12] and its distributed version HOP-TERRAIN [9].

The organization of this paper is as follows. Section 2 describes the model used in this paper. We then briefly explain MDS-MAP and HOP-TERRAIN sensor localization algorithms in section 3. Finally, section 4 states our main results.

\section{MODEL DEFINITION}

First, we assume that we have no fine control over the placement of the sensors which we call the unknown nodes (e.g., the nodes are dropped from an airplane). Formally, $n$ sensors, or unknown nodes, are distributed uniformly at random within the $d$-dimensional hypercube $[0,1]^{d}$. Additionally, we assume that there are $m$ special sensors, which we call anchors, with a priori knowledge of their own positions in some global coordinate.

Let $V_{a}=\{1, \ldots, m\}$ denote the set of $m$ vertices corresponding to the anchors and $V_{u}=\{m+1, \ldots, m+n\}$ the set of $n$ vertices corresponding to the unknown nodes. We consider the random geometric graph model $G(n, r)=$ $(V, E, P)$ where $V=V_{u} \cup V_{a}, E \subseteq V \times V$ is a set of undirected edges that connect pairs of sensors which are close to each other, and $P: E \rightarrow \mathbb{R}^{+}$is a non-negative realvalued function. We consider the function $P$ as a mapping from a pair of connected nodes $(i, j)$ to the approximate measurement for the distance between $i$ and $j$, which we call the proximity measurement. Let $\|\cdot\|$ be the Euclidean norm in $\mathbb{R}^{d}$. Define a set of random positions of $n+m$ sensors $\mathcal{X}=\left\{x_{1}, \ldots, x_{m}, x_{m+1}, \ldots, x_{m+n}\right\}$, where $x_{a} \in \mathbb{R}^{d}$ for $a \in\{1, \ldots, m\}$ is the position of anchor $a$ and and $x_{i} \in \mathbb{R}^{d}$ for $i \in\{m+1, \ldots, m+n\}$ is the position of unknown node $i$. A common model for the random geometric graph is the disc model where node $i$ and $j$ are connected if the Euclidean 


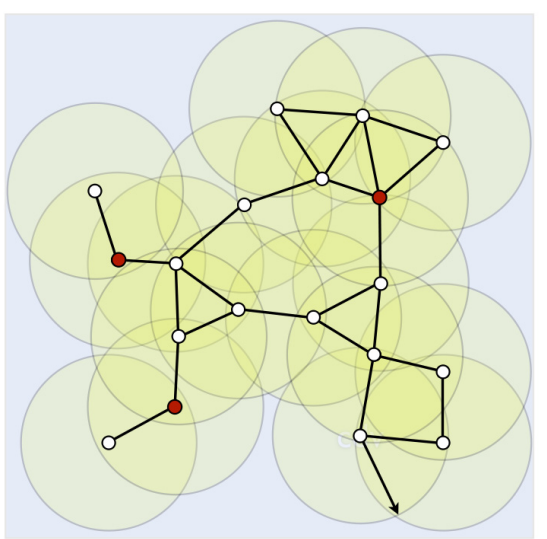

Figure 1: The disc model. Unknown nodes and anchors are denoted by white and red points respectively.

distance $d_{i, j}=\left\|x_{i}-x_{j}\right\|$ is less than or equal to a positive radio range $r$ (see Figure 1 ). To each edge $(i, j) \in E$, we associate the proximity measurement $P_{i, j}$ between sensors $i$ and $j$, which is a function of the distance $d_{i, j}$ and random noise. In an ideal case when our measurements are exact, we have $P_{i, j}=d_{i, j}$. On the other extreme, when we are given only network connectivity information and no distance information, we have constant $P_{i, j}$ 's for all $(i, j) \in E$, i.e., $P_{i, j}=r$.

In sensor network localization, distributed or localized, not all the information is available at each node. Given the graph $G(n, r)=(V, E, P)$ with associated proximity measurements for each edges in $E$, we assume that each of the nodes is aware of the proximity measurements between itself and its adjacent neighbors and each of the anchors is also aware of its own position. Moreover, communication is only possible between adjacent neighboring nodes. The goal of distributed sensor network localization is for each node to find its estimated position that best fits the measured proximity with small number of communications.

We are interested in a scalable system of $n$ unknown nodes for large value of $n$. In the unit square, assuming sensor positions are drawn uniformly at random, the graph is connected, with high probability, if $\pi r^{2}>\left(\log n+c_{n}\right) / n$ for $c_{n} \rightarrow \infty$ [2]. A similar condition can be derived for generic $d$-dimensions as $C_{d} r^{d}>\left(\log n+c_{n}\right) / n$, where $C_{d} \leq \pi$ is a constant that depends on $d$. Hence, we focus in the regime where the average number of connected neighbours is slowly increasing with $n$, namely, $r=\alpha(\log n / n)^{1 / d}$ for some positive constant $\alpha$ such that the graph is connected with high probability.

\section{LOCALIZATION}

In this section we briefly explain the centralized algorithm MDS-MAP and the distributed algorithm HOP-TERRAIN.

\subsection{MDS-MAP}

The focus of MDS-MAP when the anchors are not deployed is to find the relative map/topology of the network up to rigid transformations. Based on MDS, MDS-MAP consists of two steps :

\begin{tabular}{l}
\hline Algorithm : MDS-MAP $[12]$ \\
\hline Input: dimension $d$, graph $G=(V, E, P)$ \\
1: $\quad$ Compute the shortest paths, and let $\widehat{D}$ be \\
$\quad$ the squared shortest paths matrix; \\
2: $\quad$ Apply MDS to $\widehat{D}$, and let $\widehat{X}$ be the output.
\end{tabular}

The shortest path between node $i$ and $j$ in graph $G=$ $(V, E, P)$ is defined as a path between two nodes such that the sum of the proximity measures of its constituent edges is minimized. Let $\hat{d}_{i, j}$ be the computed shortest path between node $i$ and $j$. Then, the squared shortest paths matrix $\widehat{D} \in$ $\mathbb{R}^{n \times n}$ is defined as $\widehat{D}_{i j}=\hat{d}_{i, j}^{2}$ for $i \neq j$, and 0 for $i=j$. Note that $\widehat{D}$ is well defined only if the graph $G$ is connected. If $G$ is not connected, there are multiple configurations resulting in the same observed proximity measures and global localization is not possible.

In step 2 , we apply the MDS to $\widehat{D}$ to get a good estimate of $X$, namely, $\widehat{X}=\operatorname{MDS}_{d}(\widehat{D})$. The main step in MDS is singular value decomposition of a dense matrix $\widehat{D}$. For further information about how MDS is used in MDS-MAP please look at [7].

Note that in order to perform the second step, all the pairwise shortest paths are needed and should be sent to a central unit. This unit will then compute the MDS and find the topology of the network.

\subsection{HOP-TERRAIN}

In the distributed version, we assume that there are $n$ unknown nodes without the knowledge of their position and that there are $m$ anchor nodes with the knowledge of their position. In contrast to MDS-MAP (where the goal was to find the topology of the network not the absolute positions of each nodes) here the goal is that each node finds its global position in the network. Based on the robust positioning algorithm introduced in [9], the distributed sensor localization algorithm HOP-TERRAIN consists of two steps :

\begin{tabular}{ll}
\hline Algorithm : HOP-TERRAIN $[9]$ \\
\hline $1:$ & Each node $i$ computes the shortest paths \\
& $\left\{\hat{d}_{i, a}: a \in V_{a}\right\}$ between itself and the anchors; \\
$2:$ & Each node $i$ derives an estimated position $\hat{x}_{i}$ \\
& by triangulation with a least squares method. \\
\hline
\end{tabular}

The first step is done in the similar fashion as in MDS-MAP where each unknown nodes estimates only the distances between itself and the anchors.

In the second step, each unknown node $i \in V_{u}$ uses a set of estimated distances $\left\{\hat{d}_{i, a}: a \in V_{a}\right\}$ together with the known positions of the anchors to perform a triangulation. The resulting estimated position is denoted by $\hat{x}_{i}$. For each node, the triangulation consists of solving a single instance of a least squares problem $(A x=b)$ and this process is known as Lateration $[10,4]$.

\section{PERFORMANCE ANALYSIS}

Let $\widehat{X}$ denote an $n \times d$ estimation for $X$ with estimated position for node $i$ in the $i$ th row. Then, for MDS-MAP we need to define a metric for the distance between the original position matrix $X$ and the estimation $\widehat{X}$ which is invariant under rigid transformation of $X$ or $\widehat{X}$. Let us define $L \equiv$ $\mathbb{I}_{n}-(1 / n) \mathbb{1}_{n} \mathbb{1}_{n}^{T}$. The symmetric matrix $L$ has rank $n-1$ which eliminates the contributions of the translation, in the sense that $L X=L\left(X+\mathbb{1} s^{T}\right)$ for all $s \in R^{d}$. Note that $L$ has the following nice properties: 
- $L X X^{T} L$ is invariant under rigid transformation.

- $L X X^{T} L=L \widehat{X} \widehat{X}^{T} L$ implies that $X$ and $\widehat{X}$ are equal up to a rigid transformation.

This naturally defines following distance between $X$ and $\widehat{X}$.

$$
d_{1}(X, \widehat{X})=(1 / n)\left\|L X X^{T} L-L \widehat{X} \widehat{X}^{T} L\right\|_{F},
$$

where $\|\cdot\|_{F}$ denotes the Frobenius norm. Notice that the factor $(1 / n)$ corresponds to the usual normalization by the number of entries in the summation. Indeed this distance is invariant to rigid transformation of $X$ and $\widehat{X}$. Furthermore, $d_{1}(X, \widehat{X})=0$ implies that $X$ and $\widehat{X}$ are equal up to a rigid transformation.

Define

$$
\begin{aligned}
& r_{\mathrm{MDS}} \equiv \frac{24 d^{2}}{\sqrt{10}}\left(\frac{\log n}{n}\right)^{\frac{1}{d}} \\
& r_{\mathrm{HOP}} \equiv 8 \sqrt{3} d^{3 / 2}\left(\frac{\log n}{n}\right)^{\frac{1}{d}} .
\end{aligned}
$$

The following theorems give upper bounds on the performance of MDS-MAP and HOP-TERRAIN localization algorithms.

THEOREM 4.1. Assume that $n$ sensors are distributed uniformly at random. Then, the MDS-MAP returns estimated positions $\widehat{X}$ such that, with high probability,

$$
d_{1}(X, \widehat{X}) \leq \frac{r_{M D S}}{r}+O(r)
$$

for $r>r_{M D S}$.

ThEOREM 4.2. Assume that $n$ sensors and $m=\Omega(\log n)$ anchors are distributed uniformly at random. Then, the HOPTERRAIN returns estimated positions $\left\{\hat{x}_{i}\right\}$ such that, with high probability,

$$
\left\|x_{i}-\hat{x}_{i}\right\| \leq \frac{r_{H O P}}{r}+O(r),
$$

for $r>r_{H O P}$.

The proofs are provided in our work [3] and [7]. It is worth noting that the two bounds (2) and (3) have the same asymptotic behaviour. Since we are interested in the regime where the graph is connected (hence no isolated nodes), the first terms in upper bounds (2) and (3) are order optimum. On the other hand, since the accuracy of the measurements is $r$, we cannot avoid the second terms without any further refinement added to algorithms.

When $r=\alpha(\log n / n)^{1 / d}$ for some positive parameter $\alpha$, the error bounds in (2) and (3) are $C_{1} / \alpha+C_{2} \alpha \sqrt{\log n / n}$ for some numerical constants $C_{1}$ and $C_{2}$. The first term is inversely proportional to $\alpha$ and is independent of $n$, whereas the second term is linearly dependent on $\alpha$ and vanishes as $n$ grows large (this effect is shown numerically in Figure 2). Therefore, both MDS-MAP and HOP-TERRAIN are guaranteed to produce estimated positions less that any fixed threshold $\delta$ so long as the constant $\alpha$ and number if nodes $n$ are chosen large enough.

\section{REFERENCES}

[1] P. Biswas and Y. Ye. Semidefinite programming for ad hoc wireless sensor network localization. In IPSN '04: Proceedings of the 3rd international symposium on Information processing in sensor networks, pages 46-54, New York, NY, USA, 2004. ACM.

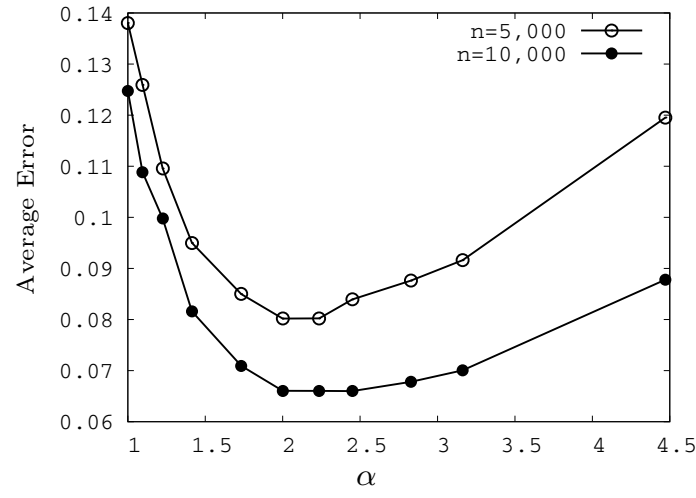

Figure 2: Numerical evaluation of upperbound (3).

[2] P. Gupta and P.R. Kumar. Critical power for asymptotic connectivity. In Proceedings of the 37th IEEE Conference on Decision and Control, volume 1, pages 1106-1110 vol.1, 1998.

[3] A. Karbasi and S. Oh. Distributed Sensor Network Localization from Local Connectivity: Performance Analysis for the HOP-TERRAIN Algorithm. In $A C M$ SIGMETRICS 2010, 2010.

[4] K. Langendoen and N. Reijers. Distributed localization in wireless sensor networks: a quantitative comparison. Comput. Netw., 43(4):499-518, 2003.

[5] R. Nagpal, H. Shrobe, and J. Bachrach. Organizing a global coordinate system from local information on an ad hoc sensor network. In IPSN '03: Proceedings of the 2nd international conference on Information processing in sensor networks, pages 333-348, 2003.

[6] D. Niculescu and B. Nath. DV based positioning in ad hoc networks. Journal of Telecommunication Systems, 22:267-280, 2003.

[7] S. Oh, A. Karbasi, and A. Montanari. Sensor network localization from local connectivity: Performance analysis for the mds-map algorithm. In 2010 IEEE Information Theory Workshop, January 6-8 2010.

[8] M. Rudafshani and S. Datta. Localization in wireless sensor networks. In IPSN '0\%: Proceedings of the 6th international conference on Information processing in sensor networks, pages 51-60, New York, NY, USA, 2007. ACM.

[9] C. Savarese, K. Langendoen, and J. Rabaey. Robust positioning algorithms for distributed ad-hoc wireless sensor networks. In USENIX Technical Annual Conference, pages 317-328, Monterey, CA, June 2002.

[10] C. Savarese, J. Rabaey, and J. Beutel. Locationing in distributed ad-hoc wireless sensor networks. In ICASSP, pages 2037-2040, 2001.

[11] A. Savvides, H. Park, and M. Srivastava. The n-hop multilateration primitive for node localization problems. Mob. Netw. Appl., 8(4):443-451, 2003.

[12] Y. Shang, W. Ruml, Y. Zhang, and M. P. J. Fromherz. Localization from connectivity in sensor networks. IEEE Trans. Parallel Distrib. Syst., 15(11):961-974, 2004.

[13] A. Singer. A remark on global positioning from local distances. Proceedings of the National Academy of Sciences, 105(28):9507-9511, 2008. 